\title{
Join-Me: Uma arquitetura para a integração entre operadores de redes móveis e provedores de serviços
}

\author{
Alexandre Martins G. de Deus ${ }^{1}$, Eduardo Pagani Julio ${ }^{1}$, Marcelo F. Moreno ${ }^{1}$ \\ ${ }^{1}$ Programa de Pós Graduação em Ciência da Computação \\ Universidade Federal de Juiz de Fora (UFJF) \\ Caixa Postal 36036-900 - Juiz de Fora - MG - Brazil \\ alexandremartins@ice.ufjf.br, eduardo.pagani@ice.ufjf.br,moreno@ice.ufjf.br
}

\begin{abstract}
The search for Quality of Experience is a constant challenge in mobile network scenarios. However, the increasing complexity of services requires an integration between service providers (SPS) and mobile network operators (MNOs). The present work proposes the Join-Me architecture, allowing components of the services of the SPs, once internalized to the MNOs infrastructure, to be managed autonomously, allowing the delivery of services as close as possible to the mobile user.
\end{abstract}

Resumo. A busca por Qualidade de Experiência é um desafio constante em cenários de redes móveis. No entanto, a crescente complexidade dos serviços demanda uma integração entre provedores de serviços (SPS) e operadores de redes móveis (MNOs). O presente trabalho propõe a arquitetura Join-Me, permitindo que componentes dos serviços dos SPs, uma vez internalizados à infraestrutura dos MNOs, sejam geridos de forma autônoma, permitindo a entrega de serviços o mais próximo possível do usuário móvel.

\section{Introdução}

Com o aumento de smartphones e de conteúdo multimídia, o tráfego de dados em redes móveis tem crescido consideravelmente, e a Qualidade de Experiência (QoE) do usuário se torna fundamental na entrega de serviços na Internet. Provedores de Serviços (SPs) terceirizam serviços de Redes de Distribuição de Conteúdo (CDNs), para descentralizar a entrega dos dados e oferecê-los mais próximo do usuário.

Operadores de Redes Móveis (MNOs) apostam na utilização de tecnologias como a Multi-access Edge Computing (MEC) para descentralizar o processamento das aplicações de rede, trazendo-o para junto das Estações Rádio Base (ERBs) e agilizando a instanciação de funções da própria rede sob demanda.

Este artigo apresenta a Join-Me, uma arquitetura integrando MNOs e SPs, objetivando permitir que componentes dos serviços dos SPs, uma vez internalizados, sejam geridos de forma autônoma e dinâmica pelos MNOs, possibilitando a entrega de serviços mais próximo do usuário. O trabalho está organizado como segue: a Seção 2 traz uma discussão sobre os trabalhos relacionados à presente proposta; a Seção 3 apresenta detalhes da arquitetura; Sua aplicabilidade por meio de Prova de Conceito é demonstrada na Seção 4. Os resultados são apresentados na Seção 5, e a 6 dedica-se à conclusão e trabalhos futuros. 


\section{Trabalhos Relacionados}

Tecnologias são criadas para lidar com requisitos de novos segmentos. A Multiaccess Edge Computing (MEC) [ETSI 2015], possibilita a redução da latência, com recursos computacionais junto às Estações Rádio Base (ERBs). Já o Network Slicing [Rost and Mannweiler 2017] no 5G [Gupta and Jha 2015], instancia segmentos virtuais de rede visando Qualidade de Serviço $(\mathrm{QoS})$ para aplicações de diferentes propósitos.

Visto que o uso de Application Program Interfaces APIs tem se tornado tendência, o Common API Framework for 3GPP Northbound APIs (CAPIF) [3rd Generation Partnership Project 2018] foi desenvolvido para padronizar e hospedar APIs de maneira centralizada.

O uso das NFVs, [Jahromi and Glitho 2018] possibilita o provisionamento de componentes de $\mathrm{CDN}$, criando servidores adicionais à rede $\mathrm{CDN}$ nas instalações do Internet Service Provider (ISP), direcionando o tráfego para o servidor mais próximo.

O conceito Follow-Me Cloud [Taleb et al. 2016], apresenta o modo que serviços em nuvem sejam migrados suavemente entre datacenters federados, sem interrupção dos mesmos e à medida que os usuários se movem, visando poupar recursos computacionais e otimizando o desempenho durante a migração. A necessidade de parte do framework ser implementada no dispositivo do usuário móvel deixa em aberto uma questão de privacidade do usuário.

Diversos trabalhos têm a melhoria da QoE do usuário como objetivo. No entanto, não há um trabalho conjunto entre i) garantir que os recursos computacionais sejam devidamente alocados; ii) dar atenção aos serviços SPs e iii) garantir a privacidade do usuário. A Join-Me visa harmonizar esses três pontos, a fim de possibilitar o trabalho conjunto dos SPs e MNOs.

\section{A arquitetura Join-Me}

É possível notar uma separação entre SPs e MNOs pois, independente dos esforços realizados por ambas as partes, toda requisição do usuário obrigatoriamente necessita percorrer o caminho pela infraestrutura do MNO até os servidores dos SPs.

Dado esse cenário, a arquitetura "Join-Me"permite que os SPs internalizem parte de seus serviços, para dentro da infraestrutura dos MNOs, por meio de uma API. Uma vez dentro da infraestrutura, o MNO se encarregará de suprir as demandas e gerenciará a rede para entregar o serviço o mais próximo possível do usuário, auxiliado por informações já presentes internamente em sua infraestrutura, como o histórico de deslocamento. Por outro lado, os Operadores de Redes Móveis poderão cobrar pelos serviços em execução e pela alocação de recursos.

Visando a privacidade do usuário, a arquitetura Join-Me se apoia na técnica de Network Slicing e no gerenciamento autônomo dos módulos para isolar aplicações. Uma vez adotado por um MNO, Join-Me pode ser suportada pela CAPIF e ter suas APIs descobertas por SPs e MNOs clientes. As APIs Join-Me não estão no escopo deste trabalho, o qual se limita à proposição da arquitetura e a sua prova de conceito. 


\subsection{Componentes da arquitetura}

Os componentes da a arquitetura Join-Me são apresentados na Figura 1. No Core da rede, a Join-Me API atua como interface entre SPs e MNOs, o Service Provider Account Manager registra novos SPs e o gerencia o controle de acesso de seus operadores. O Service Provider Authentication provê autenticação dos SPs permitindo acesso aos componentes de Join-Me. Uma vez internalizados, os módulos que compõem serviços dos SPs são armazenados no Module Repository e posteriormente o Module Orchestrator os coloca em execução. Os módulos passam a utilizar recursos dos MNOs, os quais tem sua utilização contabilizada pelo Billing Agent. As APIs da arquitetura são disponibilizadas pelo CAPIF.

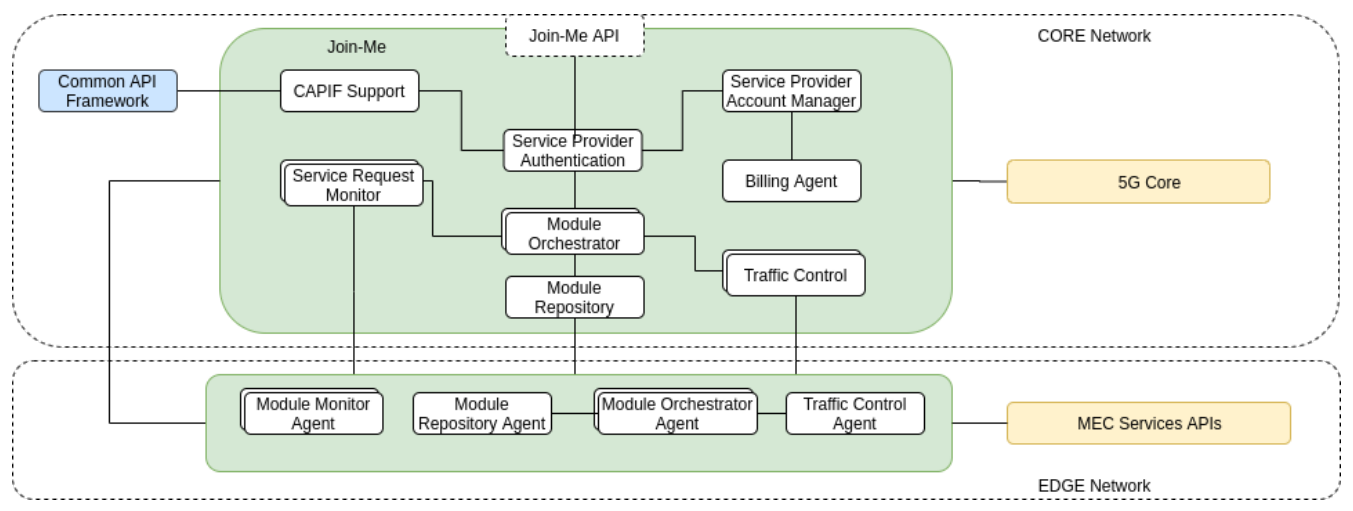

Figura 1. Arquitetura Join-Me e seus respectivos módulos

O Service Request Monitor recebe requisições dos usuários e informa a borda sobre o módulo do serviço solicitado. Ao receber tal informação, o Module Repository Agent copia do Core os módulos a serem instanciados e os armazena. O Module Orchestrator Agent disponibiliza o serviço em execução e o Traffic Control direciona as requisições para a borda. O Module Monitor Agent passa a monitorar as requisições ao serviço e, caso os recursos da borda estejam ociosos, o Traffic Control Agent redireciona as requisições de serviço de volta ao Core, onde estará disponível constantemente.

\section{Prova de Conceito}

Para demonstrar a viabilidade da arquitetura proposta, foi elaborada uma Prova de Conceito (PoC - Proof of Concept) para simular casos de uso relacionados à acomodação de serviços terceiros dentro da infraestrutura dos MNOs para as próximas gerações de redes móveis. O objetivo é demonstrar o comportamento da rede e o consumo de recursos na arquitetura. O cenário (Figura 2) consiste em um servidor para o Core da rede, duas ERBs (cada uma composta por um servidor se comportando como MEC) e por um Roteador Wi-Fi com OpenWRT ${ }^{1}$, a fim de fornecer um ponto de acesso para usuários móveis.

\subsection{Funcionamento}

Considera-se ERB um conjunto de um servidor MEC e um Access Point (AP). Assim, o servidor MEC será referenciado como ERB-MEC, enquanto o AP será ERB-AP. Assumese que os módulos dos SPs já estão em execução no servidor Core. O diagrama de sequência da Figura 3 ilustra as atividades realizadas na PoC.

\footnotetext{
${ }^{1}$ OpenWRT Project - https://openwrt.org/docs/start
} 


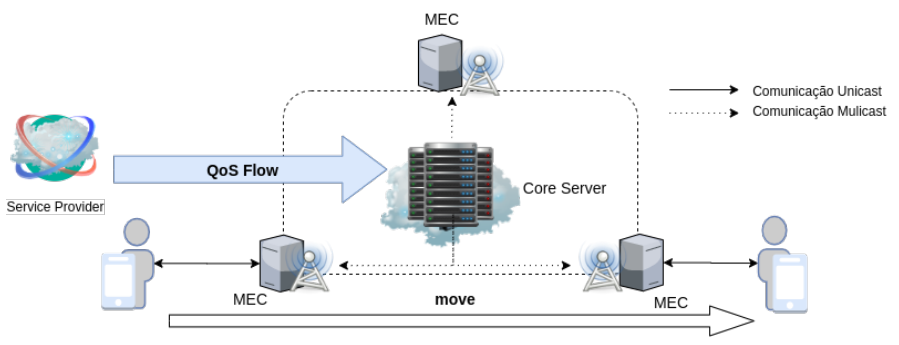

Figura 2. Cenário Prova de Conceito - Join-Me

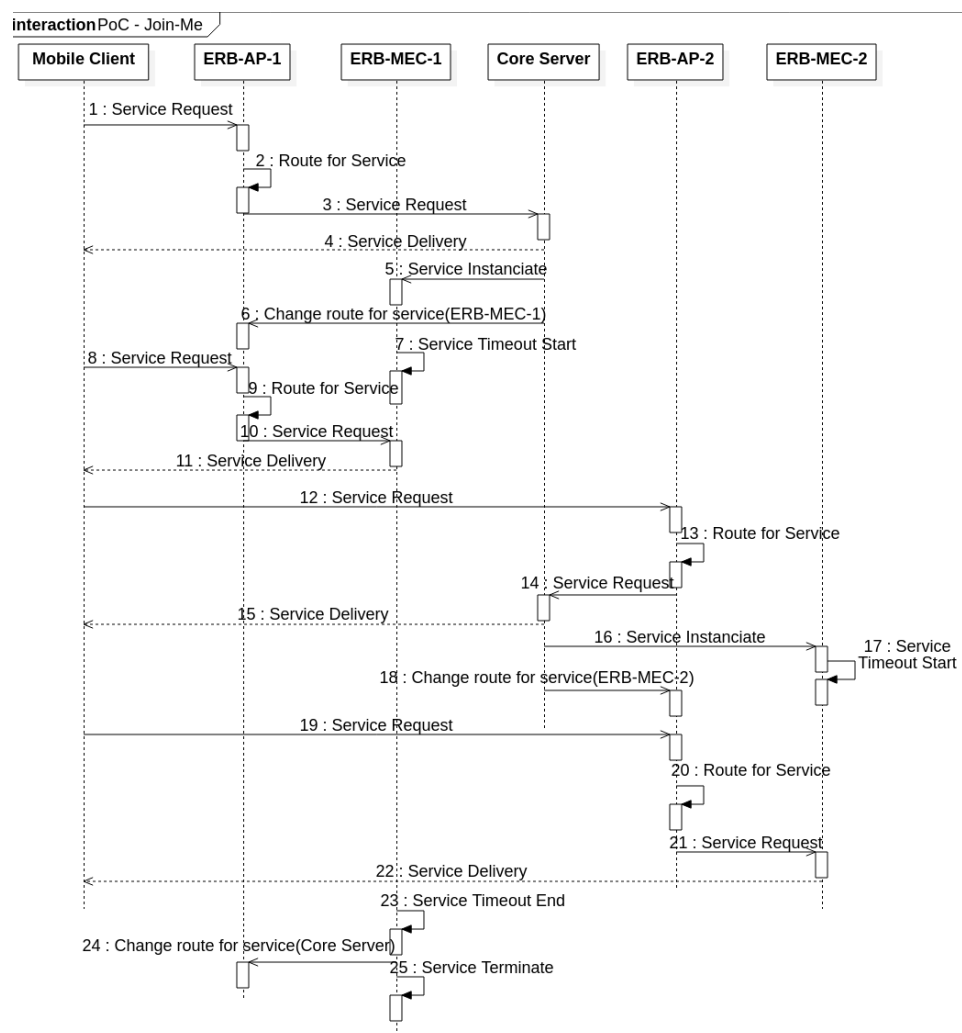

Figura 3. Diagrama de Sequência PoC - Join-Me

Considerando o "Mobile Client"conectado à ERB-AP-1, inicia-se a requisição do serviço que se encontra disponível apenas no core, conforme o passo 1 . Sendo assim, a requisição segue até o mesmo (passo 3) e a resposta é devolvida ao usuário no passo 4. O Service Request Monitor aciona o Module Orchestrator Agent para instanciar uma cópia do(s) módulo(s) na ERB-MEC-1 (passo 5). Assim que o(s) módulo(s) já esteja(m) instanciado(s) na ERB-MEC-1 (passo 6), o Traffic Control altera a rota presente na ERBAP-1 referente ao serviço requisitado para a ERB-MEC-1. Observa-se que o passo 7 é iniciado pelo Module Monitor Agent como um processo de timeout para o(s) módulo(s) instanciado(s) em ERB-MEC-1. Nos passos 8 a 11 as requisições originadas na ERB-AP1 passam a ser atendidas pela ERB-MEC-1 e não mais pelo Core.

Ao realizar o handover da ERB-AP-1 para a ERB-AP-2, o processo de requisição de serviço se repete nos passos 12 ao 22 para a sua respectiva ERB (ERB-MEC-2). Quanto a economia de recursos, percebe-se o fim do processo de timeout (passo 23) ocorrido devido à ociosidade do(s) módulo(s), uma vez que não há mais requisições originadas 
na mesma. Sendo assim, o Traffic Control Agent altera novamente a rota presente na ERBAP-1 para o servidor core e então o Module Monitor Agent aciona o Module Orchestrator Agent, que finaliza a execução do(s) módulo(s), (passos 24 e 25).

\section{Resultados}

Para a experimentação da PoC, foi elaborado um caso de uso utilizando um Streaming de vídeo ao vivo como serviço, o qual possui três módulos, o sp-live-streaming o qual envia o vídeo em um único fluxo com suas características originais por meio de Real Time Protocol (RTP) (multicast). A segmentação do vídeo é realizada pelos sp-segmentation nas resoluções 360p, 480p e 720p. Já o sp-delivery disponibiliza os segmentos ao usuário.

No experimento, o fluxo RTP multicast gerado externamente é recebido com a QoS necessária pela arquitetura, replicado-o para os segmentadores. A entrega em HTTP é realizada pelo Core da rede. Não há movimentação do usuário, observando apenas o consumo de recursos pelos módulos. Os segmentadores de resoluções superiores consomem mais recursos, uma vez que não há perda de detalhes dos frames. Focou-se apenas no tráfego HTTP, o qual está relacionado à entrega do vídeo. O sp-delivery teve seu consumo ínfimo. Como sua comunicação é Unicast, seu consumo tende a crescer linearmente em função do número de usuários, além do aumento do tráfego HTTP (Figura 4).

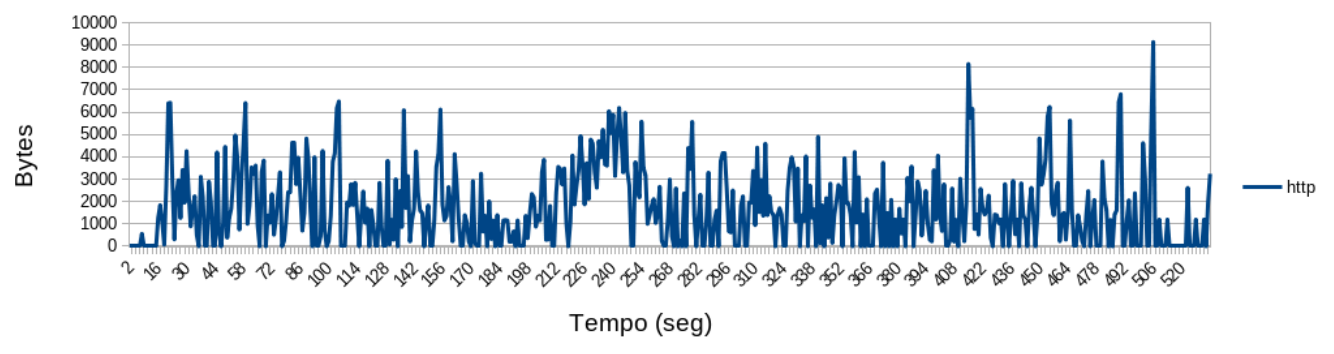

Figura 4. Tráfego HTTP - Core Join-Me

Posteriormente o serviço continuou no core da rede mas com a movimentação do usuário. Ignorou-se pequenas interrupções e observou-se que, devido a alteração no sinal wireless durante a movimentação, a perda de qualidade dos segmentos. Utilizando a PoC de Join-Me, a entrega do serviço se inicia no Core da rede e posteriormente segue todo o workflow apresentado na Figura 3. Na Figura 5 é possível observar o serviço acompanhando o usuário. Observa-se o alívio de tráfego no core da rede em comparação com o tráfego inicial do experimento (Figura 4).

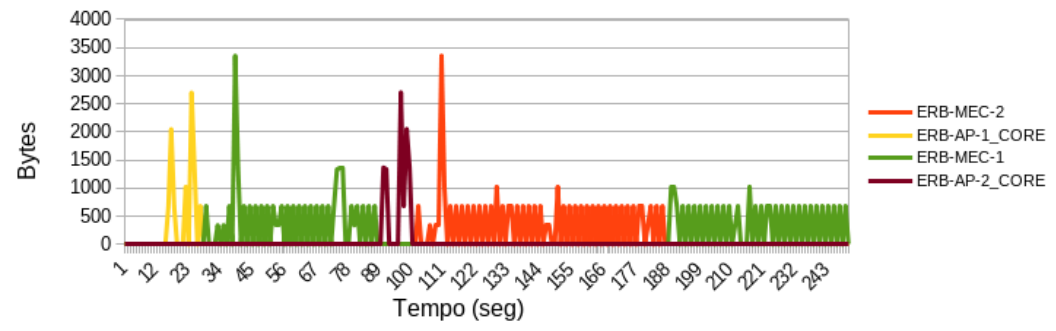

Figura 5. Tráfego HTTP e migração do serviço - Arquitetura Join-Me 


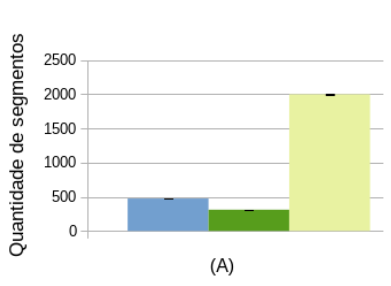

Figura 6. Análise de QoE - (A) arquitetura Join-Me
Quantidade média de seguimentos por resolução

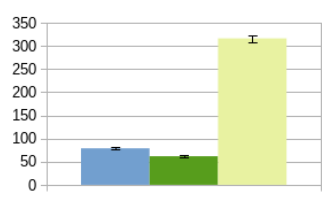

(B)
Resolução dos segmentos

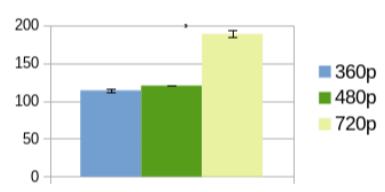

A QoE do usuário (Figura 6A), o experimento não sofreu mudanças relevantes, obedecendo o mesmo comportamento do core (Figura 6B). Se faz necessário a realização de experimentos com um maior número de usuários, para observar alterações com a elevação do tráfego HTTP. Por fim, o serviço com seus respectivos módulos foi analisado sem o suporte de Join-Me, (Figura 6C) para confrontar com os resultados da PoC. Observou-se um número maior de segmentos de baixa e média resolução em comparação a arquitetura Join-Me, devido não apenas a fatores relacionados ao sinal wireless, mas também às condições externas à rede local.

\section{Conclusão}

Este trabalho apresentou a arquitetura Join-Me, que gerencia módulos de serviços de Provedores de Serviços internalizados à infraestrutura dos Operadores de Redes Móveis. Verificou-se um ganho de QoE utilizando a arquitetura, zelando pela economia de recursos e respeitando a privacidade do usuário. Uma prova de conceito foi apresentada para exemplificar a sua utilização. Como trabalhos futuros, serão desenvolvidos os demais componentes apresentados neste trabalho e as APIs voltadas para a interação entre SPs e MNOs, incluindo o monitoramento dos serviços e recursos alocados.

\section{Agradecimentos}

Este trabalho contou com o apoio da Rede Integrada de Pesquisa em Alta Velocidade (RePesq) https://repesq.ufjf.br da Universidade Federal de Juiz de Fora.

\section{Referências}

3rd Generation Partnership Project (2018). Functional architecture and information flows to support common api framework for 3gpp northbound apis. pages 1-189.

ETSI, G. (2015). 004, mobile edge computing (mec) service scenarios v1. 1.1,(2015).

Gupta, A. and Jha, R. K. (2015). A Survey of 5G Network: Architecture and Emerging Technologies. IEEE Access, 3:1206-1232.

Jahromi, N. T. and Glitho, Roch H, e. a. (2018). An NFV and microservice based architecture for on-the-fly component provisioning in content delivery networks. In 15th IEEE Annual Consumer Communications and Networking Conference, pages 1-7.

Rost, P. and Mannweiler, Christian, e. a. (2017). Network Slicing to Enable Scalability and Flexibility in 5G Mobile Networks. IEEE Communications Magazine, 55:72-79.

Taleb, T., Ksentini, A., and Frangoudis, P. (2016). Follow-Me Cloud: When Cloud Services Follow Mobile Users. IEEE Transactions on Cloud Computing. 